\title{
Collapse phenomena during wetting in granular media
}

\author{
Fatima Zahra El Korchi ${ }^{\mathrm{a}, \mathrm{b} *}$, Frédéric Jamin ${ }^{\mathrm{a}, \mathrm{c}}$, Mohamed El Omari ${ }^{\mathrm{b}}$ and \\ Moulay Saïd El Youssoufi, \\ ${ }^{a}$ Laboratoire de Mécanique et Génie Civil, LMGC, CNRS, Université de Montpellier, France; \\ ${ }^{b}$ Laboratoire d'Automatique de l'Environnement et Procédés de Transferts, LAEPT, Université \\ Cadi Ayyad de Marrakech, Maroc;
}

${ }^{c}$ Laboratoire de Micromécanique et d'Intégrité des Structures MIST, IRSN-CNRS-Université de Montpellier, France

\begin{abstract}
We present an experimental study of identification and analysis of the collapse phenomenon in granular media, using a sample made of glass beads. The collapse potential of a soil depends on several parameters such as liquid limit, matric suction, dry apparent density, initial water content and the amount of small particles. In this study, we investigate the liquid effect during the wetting process, without external mechanical stress. Experimental tests at different initial water contents were made for different grain sizes. The equipment used is a triaxial device for unsaturated soils, instrumented with local axial and radial displacement sensors. The experimental results determined a critical water content of collapse, as well as the influence of initial water content, matric suction and grain size.
\end{abstract}

Keywords: collapse; wetting; granular media; capillarity; capillary bridge

\section{Introduction}

The collapse phenomenon can occur in unsaturated granular soils, with or without external mechanical loading. This phenomenon results from a sudden rearrangement of the solid particles during wetting. Its occurrence can affect the stability of structures, causing, for example, the differential settlement of civil engineering structures and roads, slope fractures, dykes and earth dams, landslides of underground walls or the compaction of rail network embankments.

Collapsible soils typically have a very porous and open structure. Generally, in natural soil the solid particles are bounded by deposited solutes and / or capillary bridges. When these soils are exposed to natural infiltration of water (rain, fluctuations in the level of the water table, etc.) or induced by human activity (excessive irrigation, water pipe leaks, etc.), they can undergo a collapse phenomenon.

Studies of this type of instability are often based on land observations and empirical modelling. To highlight the soil collapse phenomenon, Jennings and Knight (1957) proposed the "double oedometer test" on a sandy soil. Under oedometric mechanical loading on samples for a given initial void ratio, they concluded that wetting renders the ground unstable and is likely to trigger the collapse phenomenon. To quantify this risk, 
(a)

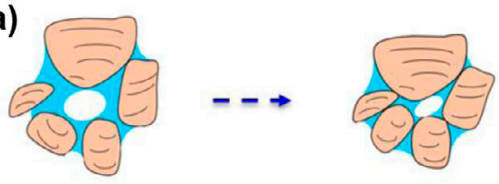

(c)
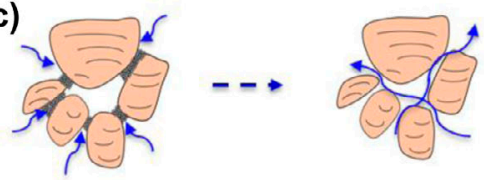

(b)

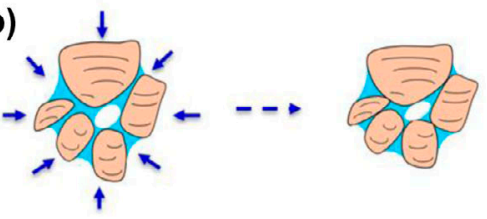

(d)

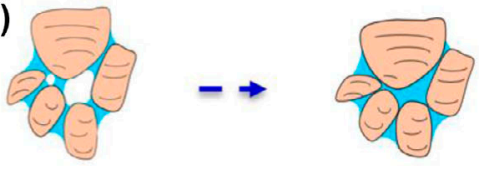

Figure 1. Mechanisms responsible for the collapse: (a) high void ratio, (b) significant applied stress, (c) cementing loss, (d) reduced matric suction.

Jennings and Knight (1975) proposed five risk levels of collapse depending on vertical deformation due to wetting under constant vertical load in oedometric testing (200 kPa).

According to the Gibbs and Barra classification (Gibbs \& Bara, 1962), liquid limit value and apparent dry density of soil are the key factors of the collapse phenomenon. Similarly, Ayadat and Ouali (1999) showed the effect of Atterberg limits (liquid limit $w_{\mathrm{L}}$ and plasticity limit $w_{\mathrm{p}}$ ) on the collapse of granular sand with different proportions of small fractions. Oedometer tests on a silty sand (Tadepalli, Rahardjo, \& Fredlund, 1992) showed that the magnitude of collapse varies linearly with the initial dry density. On the other hand, Delage, Cui, and Antoine (2005) showed that, on a silty soil collected at different depths, the amplitude of collapse is greater for high void ratio.

The effect of initial water content on the collapse magnitude, defined as the axial deformation of sample at collapse, has been a subject of several studies. Firstly, oedometer tests (Tadepalli et al., 1992), carried out on a silty soil at two different dry densities, have shown that the collapse magnitude decreases linearly with initial water content. Furthermore, other tests performed on a silty sand at various initial water contents (Delage, 2014 and Delage et al., 2005) have also shown that collapse magnitude decreases with initial water content.

In order to identify the mechanisms responsible for collapse, several researchers have linked this phenomenon to parameters such as:

- an open structure with high porosity (Barden, McGown, \& Collins, 1973) (Figure 1(a)),

- significant applied stress that tends to develop a state of instability (Barden et al., 1973) (Figure 1(b)),

- a loss of cementing, followed by migration of small particles due to water percolation (Ayadat, Belouahri, \& Ait, 1998 and Barden et al., 1973) (Figure 1(c)),

- a reduction in matric suction during wetting (Tadepalli et al., 1992) (Figure 1(d)).

However, the effect of grain size on the likelihood of collapse in granular media and the pure water process on this phenomenon remain poorly addressed experimentally.

To investigate the capillary origin of the collapse phenomenon, we use glass beads as a model. This granular material has been used in many studies and gave good results in the description and understanding of the effect of the capillary phenomena in granular media (Gras, Delenne, \& El Youssoufi, 2013; Hueckel, Mielniczuk, \& El Youssoufi, 
2013; Richefeu, El Youssoufi, \& Radjaï, 2006 and Soulié, El Youssoufi, Cherblanc, \& Saix, 2006).

A simple compression test (Soulié et al., 2006) and a direct shear test (Richefeu et al., 2006) have shown, in the pendular water domain, that granular media are sensitive to water content, which gives them an apparent cohesion through capillary action. The pendular water domain corresponds to low water contents, consequently a situation in which the capillary forces play a big part in capillary cohesion. These capillary forces are not involved in saturated state granular soils in which the behaviour is similar to that in the dry state. For intermediate states (hygroscopic, pendular and funicular), capillary action can be very variable.

Firstly, we characterised the granular media used in this study by presenting grain size distribution curves, specific densities and liquid limits. We describe the experimental apparatus and the protocol that was followed. We then present the experimental results obtained during wetting on glass beads for different grain sizes and initial water contents. Finally we discuss water effect in the collapse process of granular media.

\section{Materials}

One of the possible causes of the collapse may be the loss of capillary cohesion in granular media (Gras et al., 2013; Hueckel et al., 2013 and Soulié et al., 2006). In order to

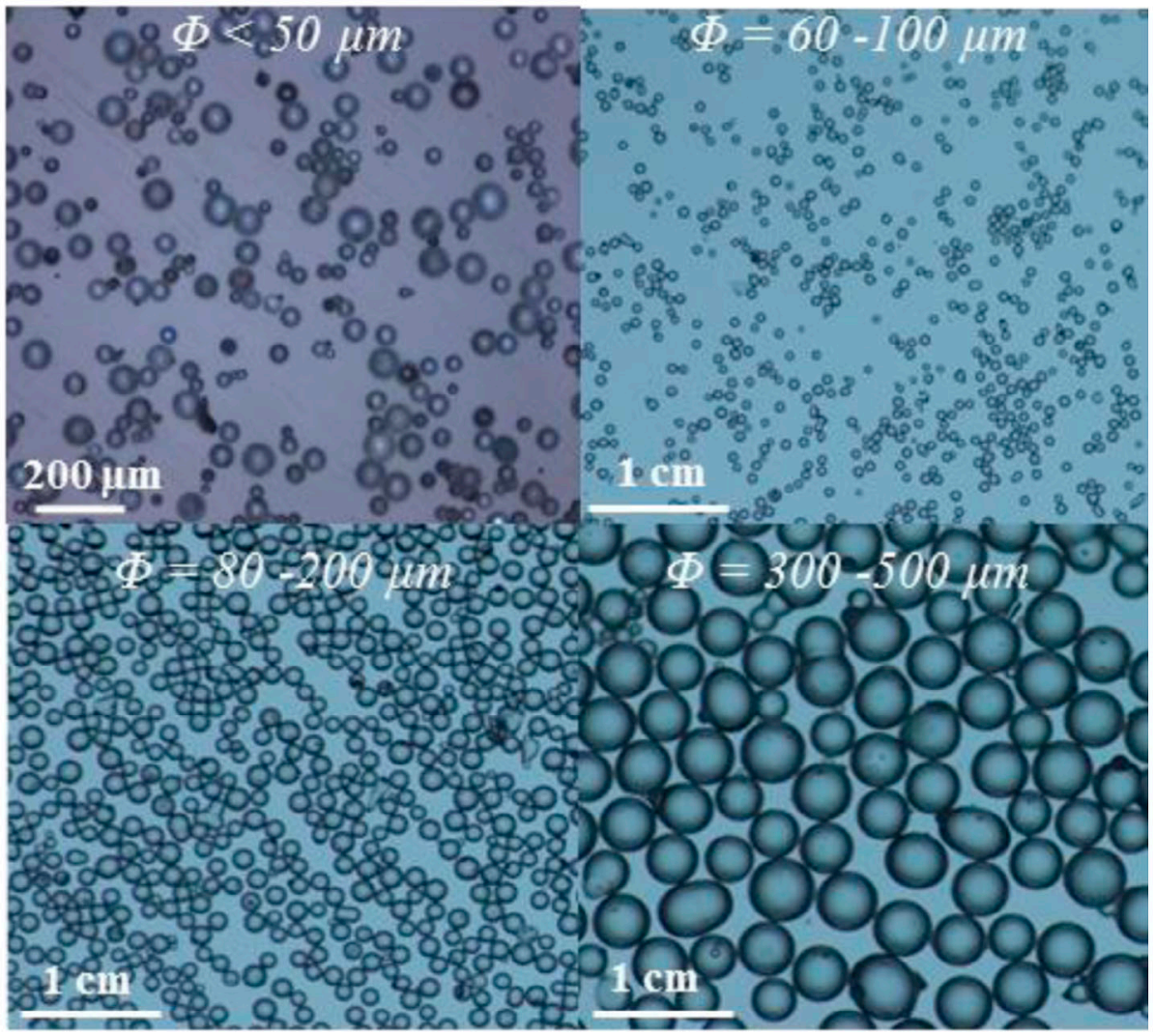

Figure 2. Sample pictures of glass beads. 


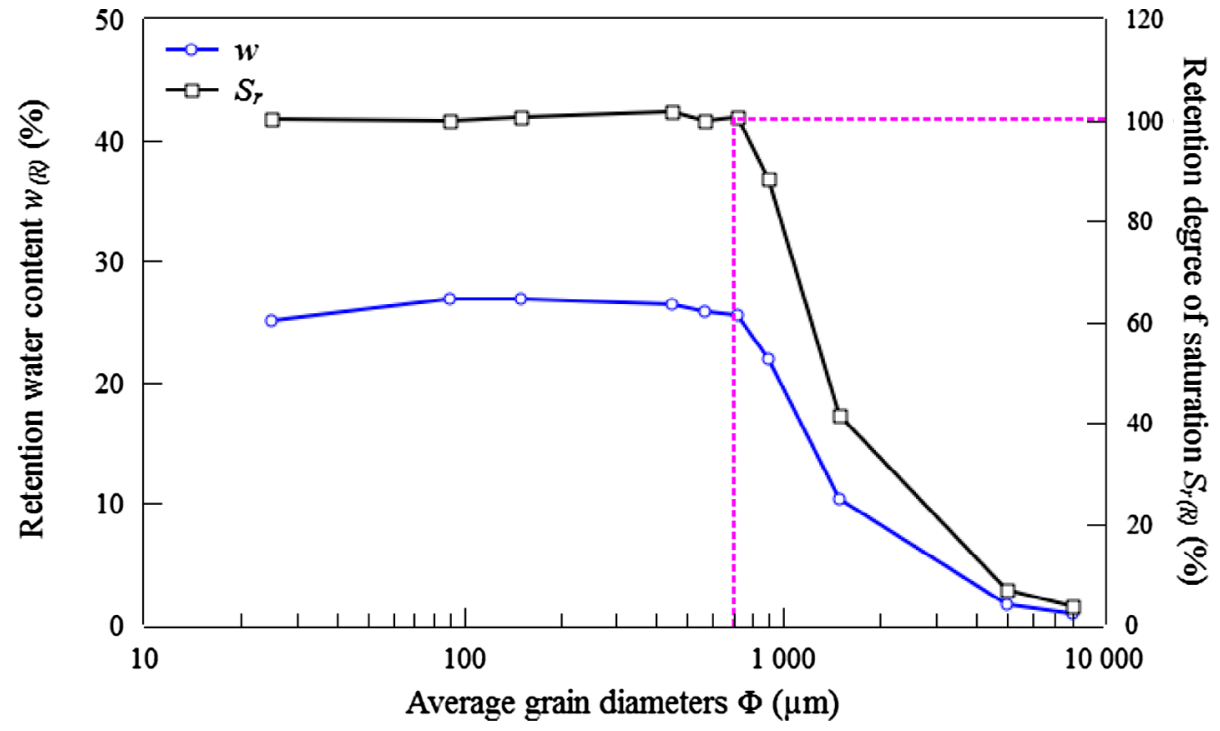

Figure 3. Retention water content $w_{(R)}$ and retention degree of saturation $S_{\mathrm{r}(\mathrm{R})}$ as a function of grain size $\Phi(c=0.59)$.

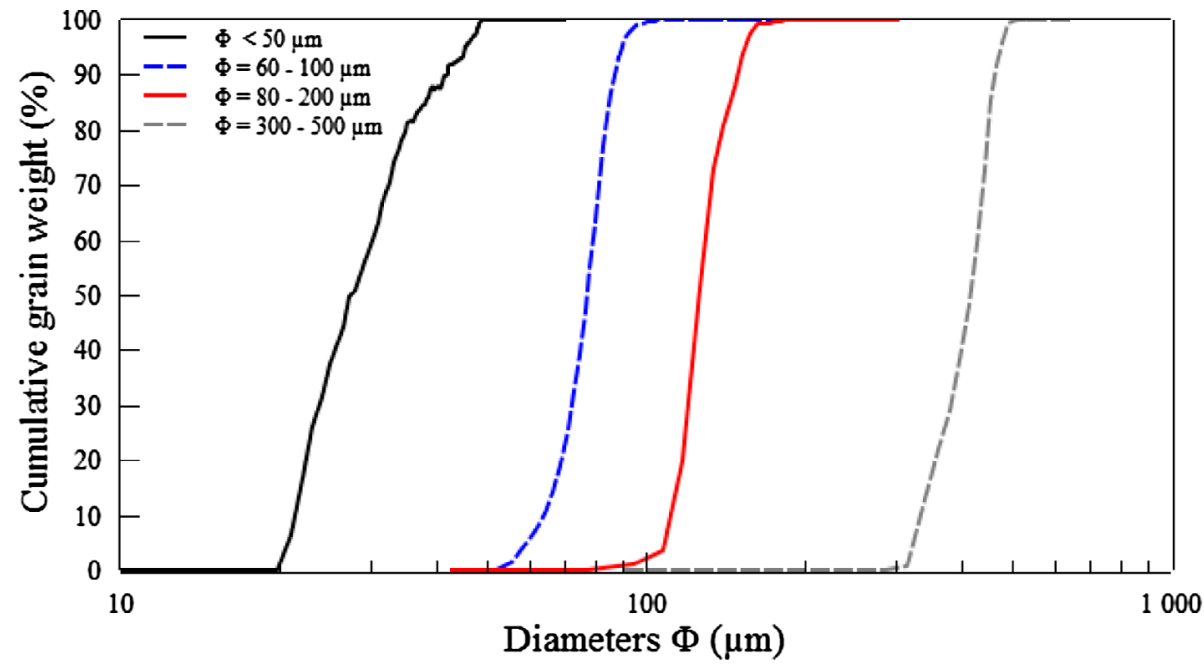

Figure 4. Cumulative grain size distribution curves of the different samples.

study this effect, we used glass beads as granular material, with different grain sizes (Figure 2). This granular media model doesn't take into account all the phenomena existing in natural soils, and more so in clayey soils. The glass beads used are substantially spherical and assumed to be non-deformable. For this type of granular media, cohesion is essentially due to the capillary bridges between the solid particles. 
Table 1. Physical properties of the different samples.

\begin{tabular}{lcccc}
\hline $\begin{array}{l}\text { Diameter } \\
\Phi(\mu \mathrm{m})\end{array}$ & $\begin{array}{c}\text { Uniformity } \\
\text { coefficient } \\
C_{\mathrm{u}}(/)\end{array}$ & $\begin{array}{c}\text { Coefficient of } \\
\text { curvature } \\
C_{\mathrm{c}}(/)\end{array}$ & $\begin{array}{c}\text { Liquid limit } \\
w_{\mathrm{L}}(\%)\end{array}$ & $\begin{array}{c}\text { Specific } \\
\text { density } \\
\rho_{\mathrm{s}}\left(\mathrm{kg} / \mathrm{m}^{3}\right)\end{array}$ \\
\hline$<50$ & 1.55 & 0.90 & $23.40 \pm 0.30$ & $2450 \pm 3$ \\
$60-100$ & 1.20 & 1.10 & $24.70 \pm 0.30$ & $2470 \pm 3$ \\
$80-200$ & 1.25 & 1.03 & $24.20 \pm 0.30$ & $2480 \pm 3$ \\
$300-500$ & 1.27 & 1.03 & - & $2500 \pm 3$ \\
\hline
\end{tabular}

In order to choose the suitable diameters for the study, the first step was to experimentally determine retention water content $w_{(\mathrm{R})}$. This water content corresponds to the soil's ability to retain water by capillary action and adsorption. The experimental test is to saturate samples for various grain sizes $(\Phi<50 \mu \mathrm{m}, \Phi=60-100 \mu \mathrm{m}, \Phi=80-200 \mu \mathrm{m}$, $\Phi=300-500 \mu \mathrm{m}, \Phi=500-630 \mu \mathrm{m}, \Phi=630-800 \mu \mathrm{m}, \Phi=800-1000 \mu \mathrm{m}, \Phi=1500 \mu \mathrm{m}$, $\Phi=5000 \mu \mathrm{m}$ and $\Phi=8000 \mu \mathrm{m})$ with compactness $c_{0}=0.59$. We placed saturated samples in a dryer where the relative humidity was about $100 \%$ to avoid drying by evaporation. The final water contents measured at equilibrium correspond to the retention water content $w_{(\mathrm{R})}$ of the granular medium (Figure 3).

For grain size less than 800 microns, Figure 3 shows that the retention water content $w_{(\mathrm{R})}$ is about $100 \%$ of saturation degree $S_{\mathrm{r}(\mathrm{R})}$. It is concluded that the capillary effect is more important in these relatively small grain sizes. Consequently, in this study, we chose four grain sizes, which are: $\Phi<50 \mu \mathrm{m}, \Phi=60-100 \mu \mathrm{m}, \Phi=80-200 \mu \mathrm{m}$ and $\Phi=300-500 \mu \mathrm{m}$. For the various sizes of beads used, the electrostatic and Van der Waals forces are negligible compared to those of capillarity (Mitchell, 1993). So, we consider that capillarity is the only source of cohesion for the used granular material.

The cumulative grain size distribution curves of the different samples chosen are determined using a photographic image treatment method (Figure 4). The uniformity coefficients $C_{\mathrm{u}}$ and the coefficients of curvature $C_{\mathrm{c}}$ of different granular media are presented in Table 1. The distribution size of glass beads chosen appears uniform and well graded. The specific density $\rho_{\mathrm{s}}$ of each grain size was also determined using the pycnometer method according to the norm NF P 94-054 (Table 1).

Liquid limit values $w_{L}$ of glass beads $\Phi<50 \mu \mathrm{m}, \Phi=60-100 \mu \mathrm{m}, \Phi=80-200 \mu \mathrm{m}$, given in Table 1, was performed using the cone penetrometer method according to the norm NF $94-052-1$ (Figure 5).

The liquid limit value of the glass beads $\Phi=60-100 \mu \mathrm{m}$ was found to be slightly higher than for grain size $\Phi=80-200 \mu \mathrm{m}$. This difference may be due to the particle size distribution of the glass beads of the two fractions. For grain size $\Phi=300-500 \mu \mathrm{m}$, liquid limit tests were not carried out because NF $94-052-1$ applies only to grain media whose diameter is less than $400 \mu \mathrm{m}$.

Figure 6 shows the retention curves for grain sizes $\Phi<50 \mu \mathrm{m}, \Phi=60-100 \mu \mathrm{m}$, $\Phi=80-200 \mu \mathrm{m}$ and $\Phi=300-500 \mu \mathrm{m}$ obtained by the filter paper technique (Whatman "42" paper). The trial was performed on samples of $60 \mathrm{~mm}$ in diameter and $30 \mathrm{~mm}$ in height with a compactness $c=0.59$. The used filter paper calibration curve is that of Hamblin (1981), where the filter paper is submitted to a wetting path during suction equilibration. Suction equilibration time was seven days in accordance with the ASTM procedure using a dry filter paper approach. A modelling of the retention curves is also plotted in Figure 6 based on Fredlund and Xing equation (Fredlund \& Xing, 1994): 


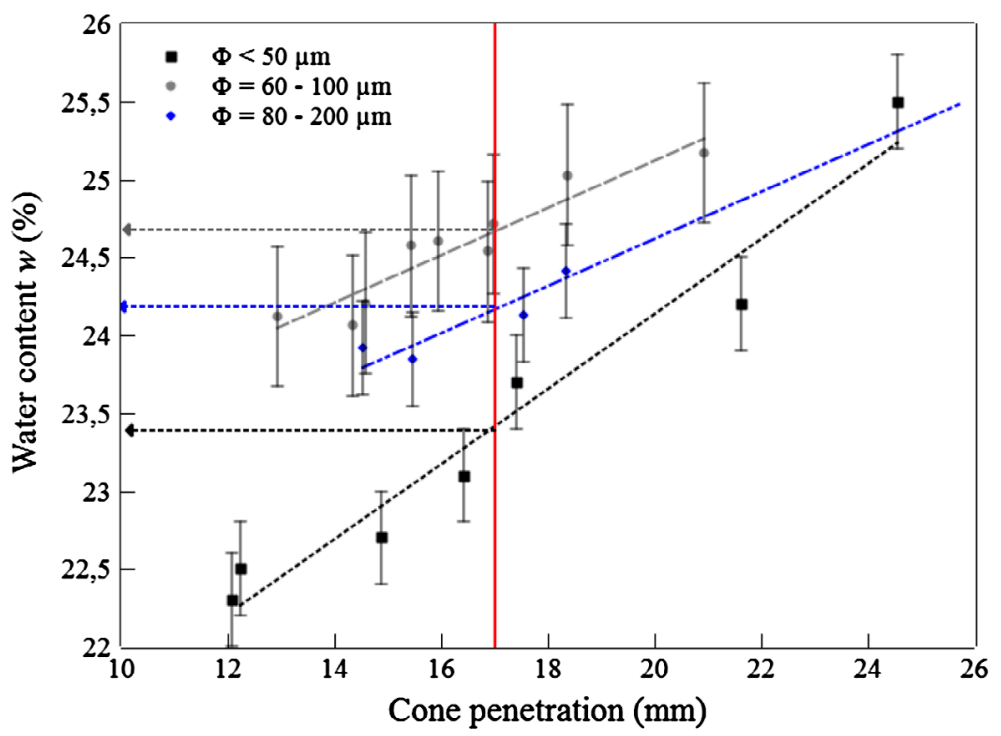

Figure 5. Liquid limit values $w_{\mathrm{L}}$ of glass beads.

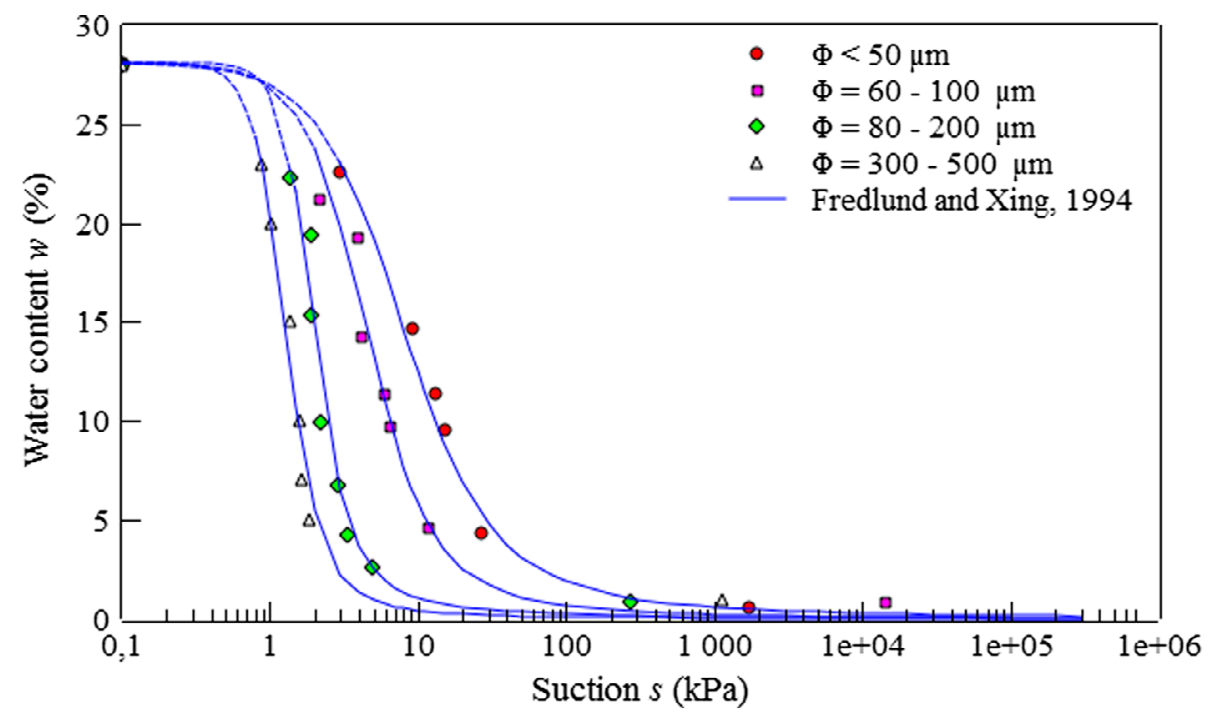

Figure 6. Retention curves for glass beads $(c=0.59)$.

$$
w=w_{\mathrm{s}} \cdot\left[\frac{1}{\ln \left(e+(s / a)^{n}\right)}\right]^{m}
$$

with $w$ being water content at different suctions $s$ and $w_{\mathrm{s}}$ saturation water content, $a, n$ and $m$ are the model parameters. 


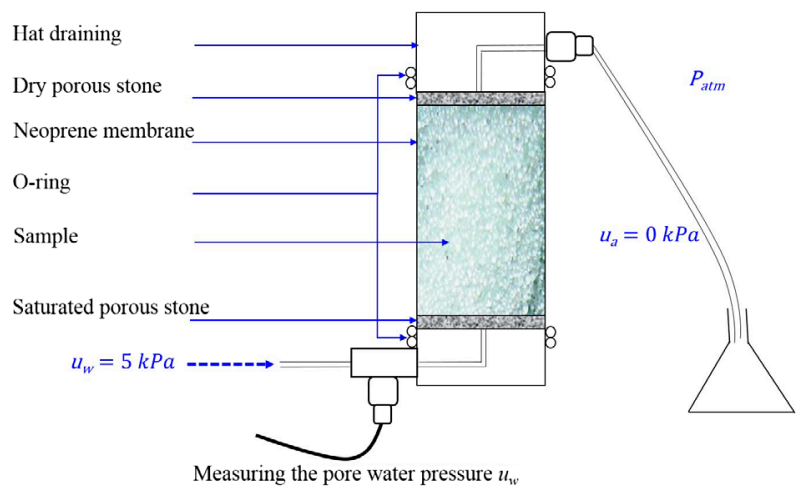

Figure 7. Illustration of wetting protocol.

(a)

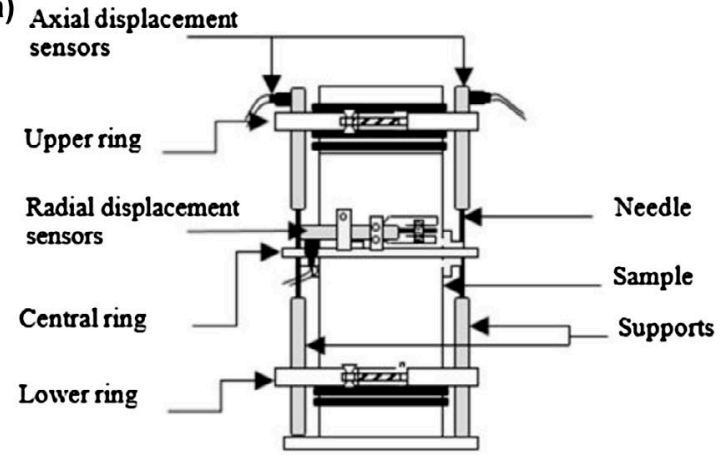

(b)

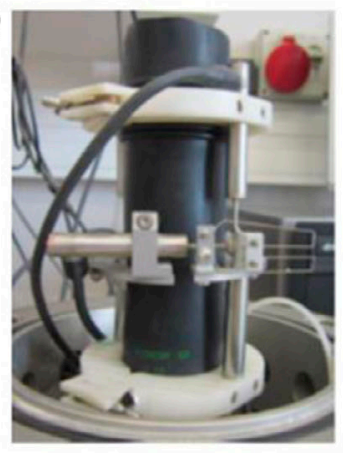

Figure 8. Illustration (a) and photograph (b) of a sample with LVDT sensors.

\section{Experimental protocol}

The glass bead samples are cylindrical in shape with an initial height $H_{0}=94 \mathrm{~mm}$ and an initial diameter $D_{0}=50 \mathrm{~mm}$. The samples were prepared with an initial compactness. $c_{0}=0.59$ and initial water contents $w_{0}=5,10$ or $15 \%$ by mixing a mass of dry glass beads $m_{\mathrm{s}}$ and a mass of deionised water $m_{\mathrm{w}}=m_{\mathrm{s}} . w_{0}$. The sample preparation is done directly in the triaxial apparatus. The wet glass beads are placed in an adapted mould surrounded with neoprene membrane. Wet glass beads are compacted in two layers using a specific device designed in our laboratory. The initial water content $w_{0}$ allows to create the capillary bridges in the sample that are at the origin of their mechanical strength by cohesion.

The wetting protocol is illustrated in Figure 7. It consists of imposing a low water pressure $\left(u_{w}=5 \mathrm{kPa}\right)$ at the bottom of the sample by the Pressure-Volume Controller while maintaining atmospheric air pressure at the top of the sample $\left(u_{\mathrm{a}}=0 \mathrm{kPa}\right)$. In these conditions, the water goes up in the sample by the capillary suction. The wetting tests were performed in the triaxial device of the type Bishop Wesley. In order to follow the axial and radial deformation of the sample, LVDT displacement sensors (both axial and one radial) were set-up as shown in Figure 8.

Axial deformations $\varepsilon_{\mathrm{a}}$ and radial deformations $\varepsilon_{\mathrm{r}}$ are deducted from the displacements measured by the local sensors according to the following relationships (Equation 2 and Equation 3): 


$$
\begin{gathered}
\varepsilon_{\mathrm{a}}=-\frac{\Delta H}{H_{0}} \\
\varepsilon_{\mathrm{r}}=-\frac{\Delta R}{R_{0}}
\end{gathered}
$$

where $\Delta H=H-H_{0}$ and $\Delta R=R-R_{0}$ are, respectively, the variations in height and radius at the sample centre (Figure 8 ). During the test, the water content $w(t)$ within the sample was determined by Equation 4:

$$
w(t)=\frac{m_{\mathrm{w}}+\Delta m_{\mathrm{w}}(t)}{m_{\mathrm{s}}}
$$

where $\Delta m_{\mathrm{w}}(t)=\rho_{\mathrm{w}} \cdot V_{\mathrm{w}}(t)$ is the mass of injected water, calculated by the water specific density $\rho_{\mathrm{w}}$ times the volume $V_{\mathrm{w}}(t)$ of injected water, directly reported by the Pressure-Volume Controller at a given time $t$ of the wetting path.

\section{Experimental results}

The wetting test results obtained on the samples of all grain size distributions $(\Phi<50 \mu \mathrm{m}, \Phi=60-100 \mu \mathrm{m}, \Phi=80-200 \mu \mathrm{m}$ and $\Phi=300-500 \mu \mathrm{m})$, with compactness $c_{0}=0.59$ and with initial water content $w_{0}=5 \%$, are shown in Figure 9. As defined, respectively, in Equations 2 and 3, a positive axial deformation corresponds to a decrease in the height of the sample and a negative radial deformation corresponds to an increase in its diameter. The measurement of pore water pressures $u_{w}$ at the bottom of samples during wetting is given in Figure 10.

During the ascent of water in the granular sample, axial deformations $\varepsilon_{\mathrm{a}}$ and radial deformations $\varepsilon_{\mathrm{r}}$ evolve gradually as shown in Figure 9. For a critical water content, deformations undergo a sudden variation assigned to the collapse phenomenon. The critical water content is subsequently called collapsing water content and noted $w_{\text {coll }}$. Figure 10 shows an increase in pore water pressure $u_{w}$ at the collapsing water content $w_{\text {coll }}$. The uncertainty of the pore water pressure sensor is $\pm 1 \mathrm{kPa}$ which justifies the small pressure fluctuation.

The collapsing water content $w_{\text {coll }}$ is defined as the water content when the capillary forces fall and no longer ensure the mechanical strength of granular material. The
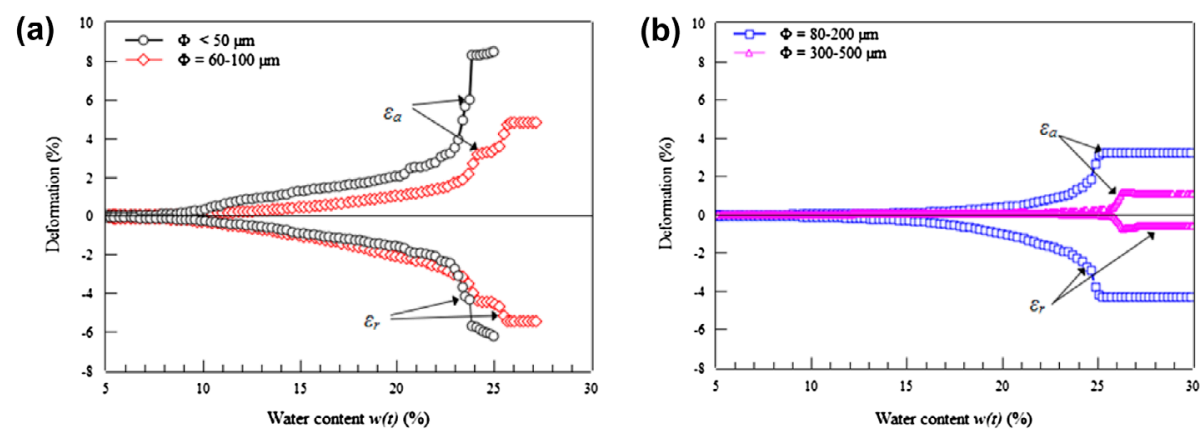

Figure 9. Evolution of axial $\varepsilon_{\mathrm{a}}$ and radial $\varepsilon_{\mathrm{r}}$ deformations during wetting for glass beads (a) $\Phi<50 \mu \mathrm{m}$, (a) $\Phi=60-100 \mu \mathrm{m}$, (b) $\Phi=80-200 \mu \mathrm{m}$ and (b) $\Phi=300-500 \mu \mathrm{m}$. 

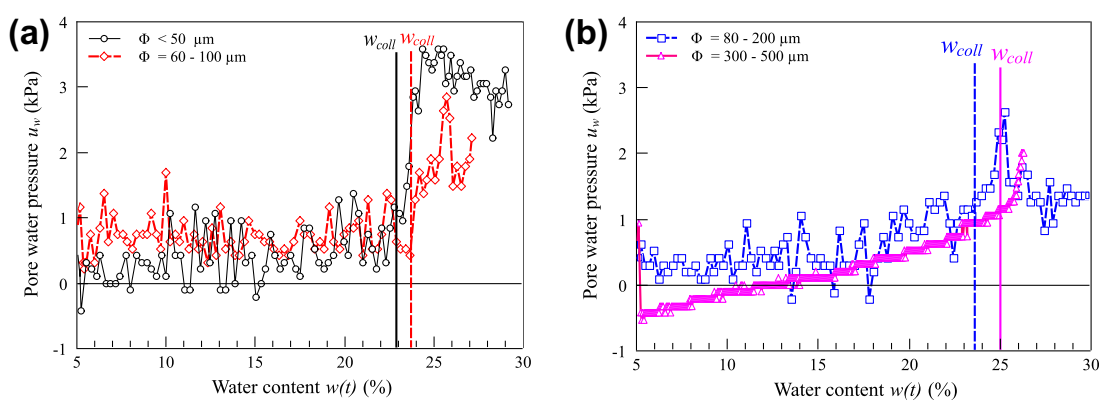

Figure 10. Evolution of water pore pressure $u_{w}$ during wetting for glass beads (a) $\Phi<50 \mu \mathrm{m}$, (a) $\Phi=60-100 \mu \mathrm{m}$, (b) $\Phi=80-200 \mu \mathrm{m}$ and (b) $\Phi=300-500 \mu \mathrm{m}$.

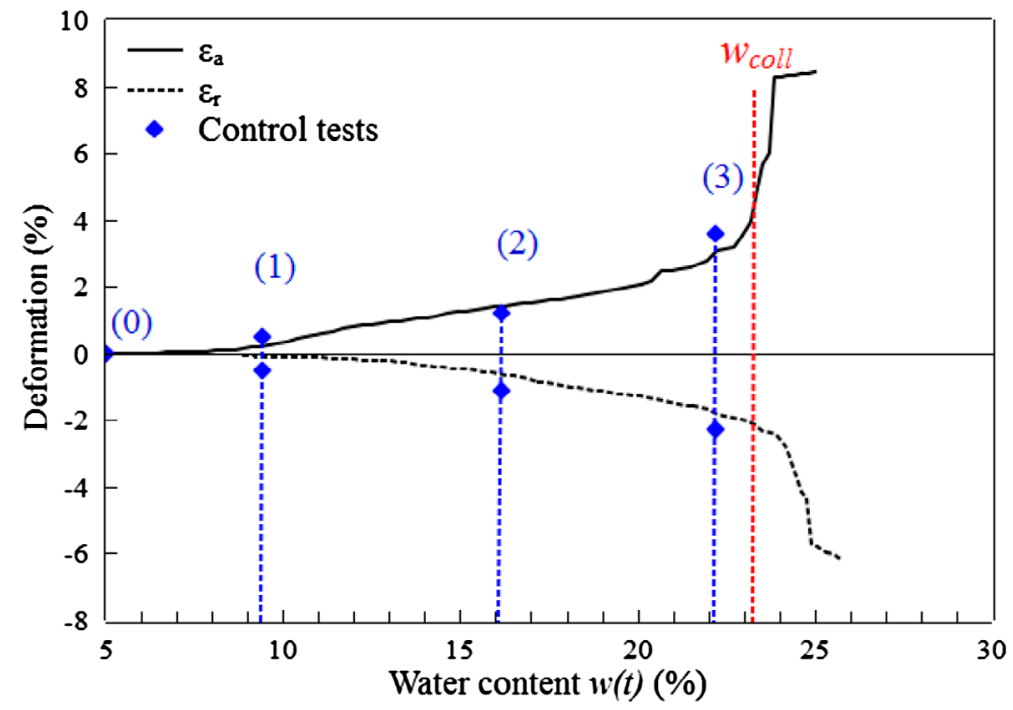

Figure 11. Evolution of axial $\varepsilon_{\mathrm{a}}$ and radial $\varepsilon_{\mathrm{r}}$ deformations during wetting for glass beads $\Phi<50 \mu \mathrm{m}$ with the three control points.

Table 2. Water content distributions in the sample during wetting.

\begin{tabular}{llcccr}
\hline \multirow{2}{*}{ Control point } & & \multicolumn{4}{c}{$w_{\text {mes }}(\%)$} \\
\cline { 3 - 6 } & & $(0)$ & $(1)$ & $(2)$ & $(3)$ \\
\hline Position & Top & 4.93 & 4.97 & 10.86 & 23.42 \\
& Middle & 4.93 & 10.90 & 20.53 & 22.25 \\
& Bottom & 4.93 & 17.21 & 21.15 & 23.02 \\
$w_{\text {avg }}(\%)$ & & 4.93 & 11.01 & 17.51 & 22.90 \\
\hline
\end{tabular}


experiments show that this fall consistently happens before the sample is saturated. In our experiments, the collapsing water content corresponds to the water content at which the axial and radial deformations are abruptly induced (Figure 9).

Experimental testing up to sample collapse provides no information about the evolution of the water content gradient. To find this gradient, three independent control tests were performed for grain size $\Phi<50 \mu \mathrm{m}$ (points (1), (2) and (3) in Figure 11). Before the occurrence of the collapse, wetting experiments were stopped at different volumes of injected water. Then, the measured water contents, denoted $w_{\text {mes }}$, were performed in an oven set at a temperature of $105^{\circ} \mathrm{C}$ on sample fractions at different positions at the top, middle and bottom (Table 2). The control tests also ensured the reproducibility of experiments.

The average of the measured water contents $w_{\text {mes }}$ at different positions of the sample, denoted $w_{\text {avg }}$, was compared to the water content $w(t)$ (Equation 4). The difference between the two values, average water contents $w_{\text {avg }}$ and water content $w(t)$, was considered as acceptable.

Table 2 shows that at the beginning of wetting an important water content gradient is set-up with higher water content at the bottom of the sample. However, just before the collapse, the water content gradient becomes negligible indicating homogenisation of the water content of any sample height.

The results of wetting tests on the different samples allow us present collapsing water content $w_{\text {coll }}$ (Figure 12(a)) and the axial deformations $\varepsilon_{\mathrm{a}}$ after collapse (Figure 12(b)) as a function of grain size.

After these results, we have been interested to identify the influence of the initial water content $w_{0}$ on the collapsing water contents $w_{\text {coll }}$, the axial $\varepsilon_{\mathrm{a}}$ and radial $\varepsilon_{\mathrm{r}}$ deformations of samples. To do so, we performed wetting tests at different initial water contents $w_{0}$ of 5, 10 and $15 \%$. The compactness of sample was $c_{0}=0.59$ for all grain sizes $(\Phi<50 \mu \mathrm{m}, \Phi=60-100 \mu \mathrm{m}, \Phi=80-200 \mu \mathrm{m}$ and $\Phi=300-500 \mu \mathrm{m})$. The results obtained on the collapsing water content $w_{\text {coll }}$ and the axial deformation $\varepsilon_{\mathrm{a}}$ after the collapse depending on initial water contents $w_{0}$ are shown in Figure 13.

Figure 14 shows the evolution of pore water pressure during wetting tests at different initial water contents $w_{0}$ of 5,10 and $15 \%$ for a sample with grain size $\Phi<50 \mu \mathrm{m}$. The other grain sizes used $(\Phi=60-100 \mu \mathrm{m}, \Phi=80-200 \mu \mathrm{m}$ and $\Phi=300-500 \mu \mathrm{m})$ show the same trends as for small granularity. As shown in the Figure 14, the collapse is always accompanied with a rough reduction in suction $\left(s=u_{\mathrm{a}}-u_{w}\right)$ because the pore

(a)

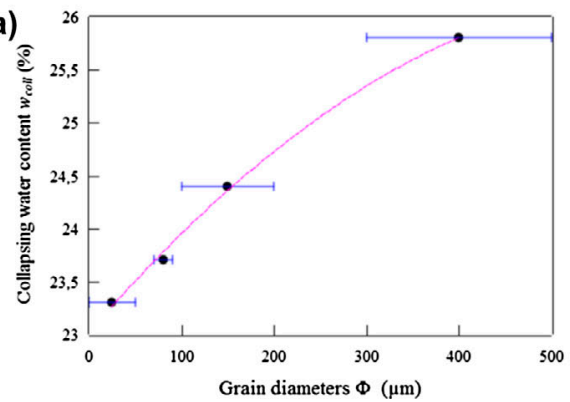

(b)

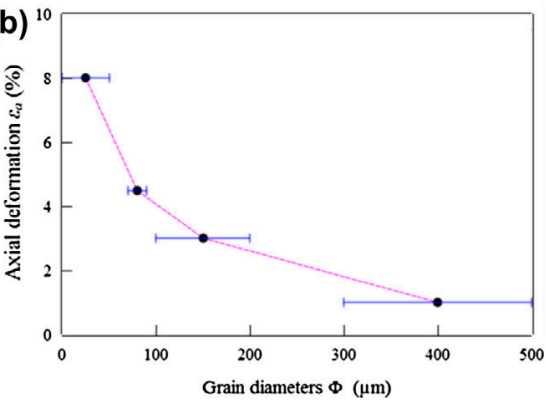

Figure 12. (a) Collapsing water content $w_{\text {coll }}$ and (b) axial deformation $\varepsilon_{\mathrm{a}}$ as a function of grain size $\Phi$. 

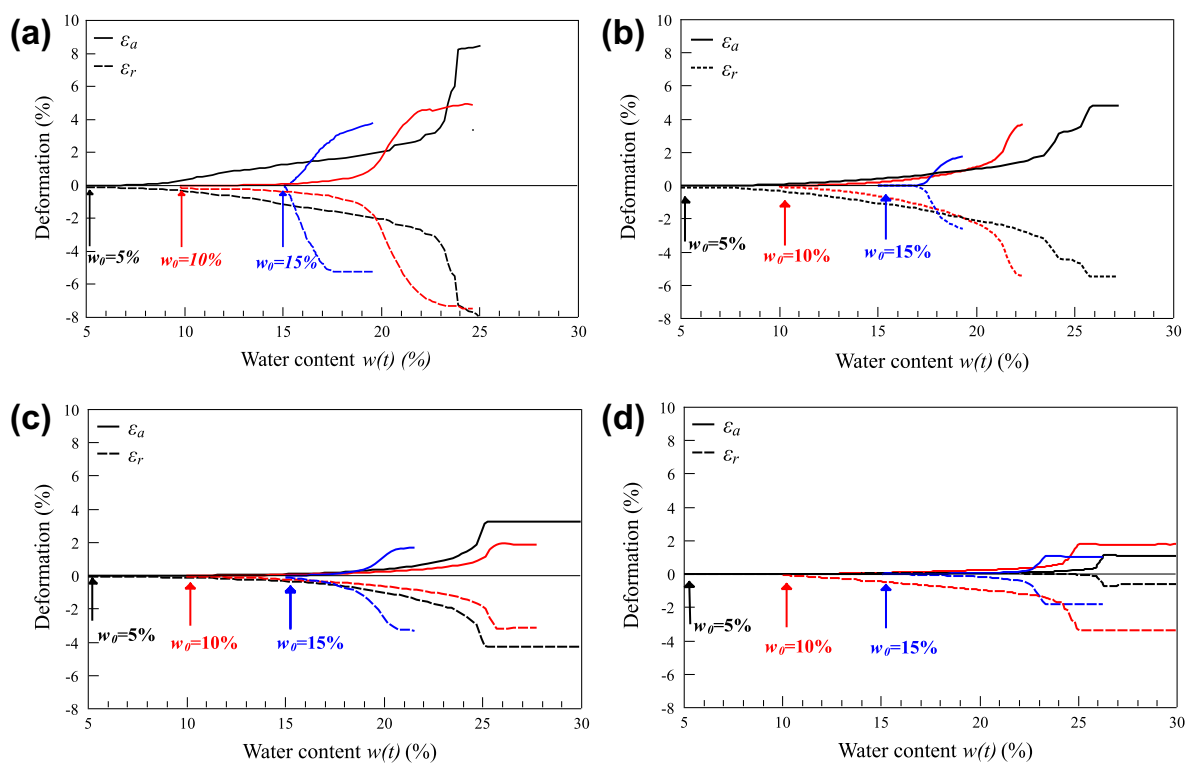

Figure 13. Effect of initial water content $w_{0}$ on deformation and collapsing water content $w_{\text {coll }}$ during wetting ((a) $\Phi<50 \mu \mathrm{m}$, (b) $\Phi=60-100 \mu \mathrm{m}$, (c) $\Phi=80-200 \mu \mathrm{m}$ and (d) $\Phi=300-500 \mu \mathrm{m}$ ).

water pressure $u_{w}$ increases suddenly at the time of the collapse. During the test, the pore air pressure $u_{\mathrm{a}}$ is equal to zero in effective pressure corresponding to the atmospheric pressure. When the macroscopic pore water pressure is well defined (funicular domain), suction can be written as follows:

$$
s=-u_{w}
$$

These results show a dependence of the collapsing water content $w_{\text {coll }}$ on initial water content $w_{0}$ of the granular medium where there is an almost linear decrease (Figure 15(a)). This dependence is more pronounced for small grain size $\Phi<50 \mu \mathrm{m}$ and $\Phi=60-100 \mu \mathrm{m}$. Figure 15(b) shows that the initial water content of the samples had little effect on the axial deformation amplitudes for grain sizes greater than $100 \mu \mathrm{m}$.

\section{Discussion}

Wetting tests were performed on glass bead samples without confining stress $\left(\sigma_{3}=0 \mathrm{kPa}\right)$ to highlight the original collapse mechanisms, which are purely waterborne. Wetting in a granular medium induced dimensional changes in the cylindrical sample. The deformations due to wetting took an abrupt form at a critical water content, called collapsing water content $w_{\text {coll }}$, for which the sample instantly lost its mechanical strength (Figure 9). This figure shows that the increase in water content involves an increase in deformations as a result of the decrease in cohesion. For low water contents, the capillary cohesion increases with the water content as shown by Richefeu et al., 2006; Soulié et al., 2006. For high water contents, the capillary bridges merge and the liquid phase becomes continuous in the sample. In this state, the capillary cohesion decreases with higher water contents and seems to be the origin of the observed collapse. The loss of mechanical strength was 

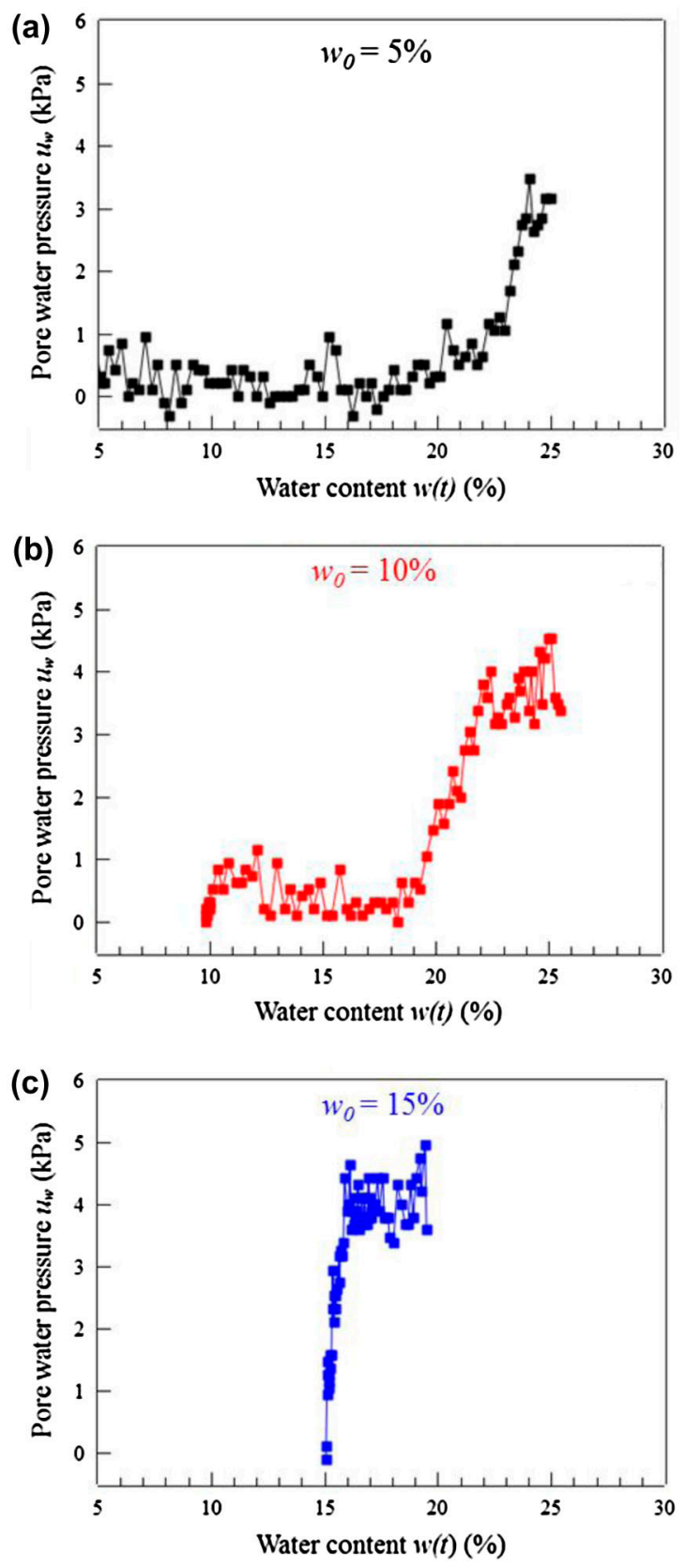

Figure 14. Evolution of the pore water pressure $u_{w}$ for different initial water contents $w_{0}$ : (a) $w_{0}=5 \%$, (b) $w_{0}=10 \%$ and (c) $w_{0}=15 \%$ (Glass beads $\Phi<50 \mu \mathrm{m}$ ).

accompanied by an increase in pore water pressure within the sample, due to a rapid rearrangement of the grains (Figures 10 and 14).

For low initial water content $\left(w_{0}=5 \%\right)$, it was noted that the collapsing water content $w_{\text {coll }}$ (for grain sizes: $\Phi<50 \mu \mathrm{m}, \Phi=60-100 \mu \mathrm{m}$ and $\Phi=80-200 \mu \mathrm{m}$ ) was 

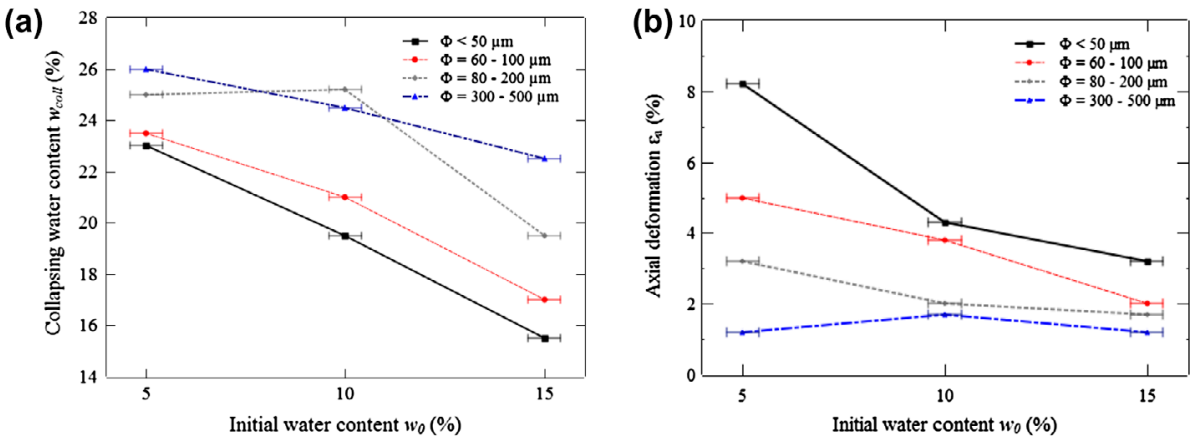

Figure 15. (a) Effect of initial water content $w_{0}$ on the collapsing water content $w_{\text {coll }}$ and (b) on axial deformation $\varepsilon_{\mathrm{a}}$ of samples.

approximately equal to the liquid limit $w_{\mathrm{L}}$ measured by the penetration cone method (Figures 5 and 12(a)). This parameter $\left(w_{\mathrm{L}}\right)$ has also been used by several authors to characterise collapsible soils (Ayadat \& Ouali, 1999 and Gibbs \& Bara, 1962).

Collapsing water content $w_{\text {coll }}$ and amplitude deformation, particularly for axial deformations $\varepsilon_{\mathrm{a}}$ (Figure 12) depended on grain size. We can see that the collapsing water content $w_{\text {coll }}$ increased with grain size. Axial deformation $\varepsilon_{\mathrm{a}}$, decreased non-linearly with grain size. Both results show the high sensitivity of small granularity samples to wetting. This sensitivity can be explained by the different microstructural morphology of the samples, having the same volume fraction. The finer the grain size, the more dominant are the capillary forces relative to the gravity effect (Mitchell, 1993). The increase in the water content leads to a progressive merger of capillary bridges and subsequently a reduction in capillary forces that impacts more the finer grain. This effect is illustrated on the Figure 12(b) where we can notice that the fine grains present higher axial deformation.

For the wetting tests with different initial water content $w_{0}(5,10$ and $15 \%)$, the critical water content $w_{\text {coll }}$ was seen to decrease with the initial water content $w_{0}$ (Figure 15(a)). This decrease was even more significant for small grain size (Figure 13), which appeared to be directly related to changes in matric suction (Figure 6).

The evolution of suction with different water contents $w(5,10$ and $15 \%)$, showed the suction decrease to be more important for the smaller grain sizes $(\Phi<50 \mu \mathrm{m}$ and $\Phi=60-100 \mu \mathrm{m})$ than the coarser media $(\Phi=80-200 \mu \mathrm{m}$ and $\Phi=300-500 \mu \mathrm{m})$ (Figure 6). This relation between collapse and suction decrease is similar to the explanation of the collapse mechanism proposed by Tadepalli et al. (1992). It is also noted that axial deformation after the collapse is higher when the initial water content is low (Figures 13 and 15 (b)). This effect of initial water content on the amplitude of the collapse is similar to the results in the literature on mechanical stress inducing a collapse on sandy loam soil type (Tadepalli et al., 1992) and silty type of grain size between 2 and $80 \mu \mathrm{m}$ (Delage, 2014 and Delage et al., 2005).

\section{Conclusion}

The collapse phenomenon observed on samples of glass beads comes from the loss of cohesion subsequent to the progressive merger of capillary bridges during wetting. Therefore, one possible origin of the collapse phenomenon on sandy soils is the loss of 
capillary cohesion. However, in the case of clayey soils, this effect is associated with other more complex phenomena.

This phenomenon occurs at a critical water content with a notable decrease in the suction within the sample. For this critical water content, the capillary forces decrease enough to reduce the mechanical strength of the sample and this effect is more important on the axial deformation for finer grain. The effect of grain size particle and initial water content were highlighted. The high sensitivity of granular media to collapse can be explained by the morphological description of the samples (number of contacts, capillary bridges and pore distribution) and the reduction in matric suction with wetting. The water content at which the collapse occurs for initial water content $w_{0}=5 \%$ is very close to the liquid limit of studied samples. Unfortunately, this does not seem to be the case for higher initial water contents $\left(w_{0}=10 \%\right.$ and $\left.w_{0}=15 \%\right)$.

\section{Disclosure statement}

No potential conflict of interest was reported by the authors.

\section{Funding}

This work was supported by Labex NUMEV "Laboratoire d'Excellence : Solutions Numériques, Matérielles et Modélisation pour l'environnement et le vivant", France and CNRST "Centre National pour la Recherche Scientifique et Technique" [Scholarship number : I003/013], Morocco.

\section{References}

Ayadat, T., Belouahri, B., \& Ait, Ammar R. (1998). Migration of fine size particles as a collapse mechanism of soils. Revue Française de Géotechnique, 83, 73-83.

Ayadat, T., \& Ouali, S. (1999). Identification des sols affaissables basée sur les limites d'Atterberg. Revue Française de Géotechnique, 86, 53-56.

Barden, L., McGown, A., \& Collins, K. (1973). The collapse mechanism in partly saturated soil. Engineering Geology, 7, 49-60.

Delage, P., Cui, Y. J., \& Antoine, P. (2005). Geotechnical problems related with loess deposits in northern France. Proceedings of International Conference on Problematic Soils, Famagusta, Cyprus. Bilsel and Nalbantoglu (eds). 2, 517-540.

Delage, P. (2014). Geotechnical problems due to the collapse of unsaturated soils: the case of loess from northern France. Journal of Applied Science \& Engineering Technology, 1, 17-22.

Fredlund, D. G., \& Xing, A. (1994). Equations for the soil-water characteristic curve. Canadian Geotechnical Journal, 31, 521-532.

Gibbs H. J., \& Bara J, P. (1962). Predicting surface subsidence from basic soil tests. Special Technical Publication $N^{\circ}$ 322, American Society for Testing and materials ASTM Special Technical Publication, 322, 277-283.

Gras, J.-P., Delenne, J.-Y., \& El Youssoufi, M. S. (2013). Study of capillary interaction between two grains: a new experimental device with suction control. Granular Matter, 15, 49-56.

Hamblin, A. P. (1981). Filter-paper method for routine measurement of field water potential. Journal of Hydrology, 53, 355-360.

Hueckel T., Mielniczuk B., \& El Youssoufi M. S. (2013, March). Micro-scale study of rupture in desiccating granular media. Paper presented at the Geotechnical Special Publication GSP 231, Geo-Congress 2013, San Diego, pp. 808-817, doi:10.1061/9780784412787.082.

Jennings J. E., \& Knight K. (1957). The additional settlement of foundation due to a collapse of structure of sandy subsoils on wetting. Proceeding of the 4th International Congress on Soil Mechanics and Foundation Engineering, London, 1, 316-319.

Jennings J. E., \& Knight K. (1975). A guide to construction on or with materials exhibiting additional settlement due to collapse of grain structure. Proceeding of the 6th Regional Conference for Africa on Soil Mechanics and Foundation Engineering, Durban, South Africa, 1, 99-105. 
Mitchell, J. K. (1993). Fundamentals of soil behavior (2nd ed.). New York: Wiley Inter Science.

Richefeu, V., El Youssoufi, M. S., \& Radjaï, F. (2006). Shear strength properties of wet granular materials. Physical Review E, 73, 051304.

Soulié, F., El Youssoufi, M. S., Cherblanc, F., \& Saix, C. (2006). Capillary cohesion and mechanical strength of polydisperse granular materials. The European Physical Journal E, 21, 349-357.

Tadepalli, R., Rahardjo, H., \& Fredlund, D. G. (1992). Measurement of matric suction and volume changes during inundation of collapsible soils. Geotechnical Testing Journal, 15, 115-122. 